\title{
Anaphylaxis induced by hydroxyethyl starch during general anesthesia
} -A case report-

\author{
Hyun Jee Kim, Sae Young Kim, Min Ju Oh, and Jin Mo Kim \\ Department of Anesthesiology and Pain Medicine, School of Medicine, Keimyung University, Daegu, Korea
}

Hydroxyethyl starch (HES) solutions are synthetic non-protein colloid solutions used to treat hypovolemia. However, their use is not free from the risk of allergic reactions. A 42-year-old male was scheduled to undergo aortic-iliacfemoral bypass surgery for the treatment of arteriosclerosis obliterans. He had no history of allergy. Two hours after the start of surgery, and within minutes after HES administration, facial erythema, hypotension and bronchospasm developed. HES infusion was discontinued under the estimation of anaphylaxis. The patient received phenylephrine, ephedrine, diphenhydramine and hydrocortisone with hydration. After restoration of vital signs, surgery was performed without complications. (Korean J Anesthesiol 2012; 63: 260-262)

Key Words: Anaphylaxis, Bronchospasm, Erythema, Hydroxyethyl starch.

Hydroxyethyl starch (HES) solutions are a widely used plasma substitute for correcting intraoperative hypovolemia. HES refers to a class of synthetic non-protein colloid solutions that are modified natural polysaccharides and are similar to glycogen and derived from amylopectin, consisting of hydroxyethylated polymers of glucose. HES preparations are defined by concentration, molar substitution, molar substitution ratio and average molecular weight [1].

The possible side effects of HES are allergic reactions, alterations of the hemostasis resulting in increased bleeding, renal failure, tissue storage and pruritus. Allergic reaction induced by HES is caused by the substance itself (starch) [2], and the incidence of allergic reactions is lowest compared with that of other colloids [3]. There was only one case of anaphylactic reaction probably induced by HES, reported so far in Korea [4].

Herein, we present a patient who developed a severe intraoperative anaphylactic reaction soon after initiation of HES 130/0.4 infusion, along with a review of the literature.

\section{Case Report}

A 42-year-old male (weight $71 \mathrm{~kg}$; height $172 \mathrm{~cm}$ ) was scheduled to undergo aortic-iliac-femoral bypass surgery for

Received: May 9, 2011. Revised: June 22, 2011. Accepted: August 2, 2011.

Corresponding author: Sae Young Kim, M.D., Department of Anesthesiology and Pain Medicine, School of Medicine, Keimyung University, 194, Dongsan-dong, Jung-gu, Daegu 700-712, Korea. Tel: 82-53-250-7287, Fax: 82-53-250-7240, E-mail: mandell@dsmc.or.kr

@) This is an open-access article distributed under the terms of the Creative Commons Attribution Non-Commercial License (http:// creativecommons.org/licenses/by-nc/3.0/), which permits unrestricted non-commercial use, distribution, and reproduction in any medium, provided the original work is properly cited. 
the treatment of arteriosclerosis obliterans (ASO). Other than smoking a pack of cigarettes per day for 20 years, the patient's past medical history revealed no unusual findings. There was no history of allergy, either for the patient himself or his family. There were no abnormalities in the preoperative physical examination, hematologic tests, simple X-ray test and ECG.

Thirty minutes before arriving in the operation room, $3 \mathrm{mg}$ of midazolam and $0.2 \mathrm{mg}$ of glycopyrrolate were administrated intramuscularly as premedication. Pre-anesthetic blood pressure (BP), heart rate (HR) and pulse oximeter oxygen saturation $\left(\mathrm{SpO}_{2}\right)$ were 105/55 $\mathrm{mmHg}, 81$ beats/min (bpm) and 99\%, respectively. Endotracheal intubation was performed in the supine position, 1 minute after the IV administration of $12 \mathrm{ml} \mathrm{1 \%} \mathrm{propofol} \mathrm{(Pofol} \mathrm{inj,} \mathrm{Dongkook} \mathrm{Co.,} \mathrm{Republic} \mathrm{of}$ Korea) and $50 \mathrm{mg}$ rocuronium (Esmeron ${ }^{\circledR}$, NV Organon, Netherlands). A mixture of sevoflurane air $2 \mathrm{~L} / \mathrm{min}$ and $\mathrm{O}_{2} 1 \mathrm{~L} /$ min was then delivered for maintenance of anesthesia under volume controlled ventilation, at which the peak inspiratory pressure (PIP) was $18 \mathrm{mmHg}$ and the end tidal carbon dioxide concentrations $\left(\mathrm{EtCO}_{2}\right)$ were between 35 and $40 \mathrm{mmHg}$. A radial artery catheter was inserted to continuously monitor the arterial BP. During the first 2 hours after the start of surgery, the vital signs were stable, but the urine output was not sufficient. Then, HES 130/0.4 (Voluven ${ }^{\circledR}$, Fresenius Kabi, Bad Homburg, Germany) was administered through a peripheral i.v. line for volume therapy. About 5 minutes from the time when HES administration was started, erythema was observed in the face and neck, with normal body temperature. Within about 10 minutes of initiation of the HES infusion, and after approximately $200 \mathrm{ml}$ were given, arterial BP suddenly dropped to $70 / 35 \mathrm{mmHg}$, and the $\mathrm{HR}$ was increased to $115 \mathrm{bpm}$. $\mathrm{SpO}_{2}$ was reduced to $92 \%$ with a high PIP ( $36 \mathrm{mmHg}$ ). Capnography showed an obstructive pattern and $\mathrm{EtCO}_{2}$ decreased abruptly from $35 \mathrm{mmHg}$ to $22 \mathrm{mmHg}$. Assessment of the endotracheal tube, breathing circuit and anesthetic machine revealed no evidence of mechanical obstruction. On auscultation, mild wheezing was noted in both lung fields. All signs, including erythema, hypotension and bronchospasm, fulfilled the clinical criteria for diagnosing anaphylaxis. Because antibiotics or blood components were not used during the operation, rocuronium was injected before 70 minutes without any problem and the anaphylactic reaction occurred within minutes of HES exposure, HES was suspected as the culprit and its infusion was therefore discontinued. The patient's ventilation was switched to $100 \% \mathrm{O}_{2}$ and multiple boluses of phenylephrine (cumulative dose 600 $\mu \mathrm{g}$ IV) and ephedrine (8 mg IV increments) were administered, in conjunction with isotonic crystalloid. Sevoflurane concentration was continuously maintained at 1 MAC during the event. The arterial blood gas analysis showed $\mathrm{pH}$ 7.31, $\mathrm{PCO}_{2}$ $48 \mathrm{mmHg}, \mathrm{PO}_{2} 82 \mathrm{mmHg}, \mathrm{HCO}_{3}{ }^{-} 22.7 \mathrm{mmol} / \mathrm{L}$ and $\mathrm{BE}-3.6$ $\mathrm{mmol} / \mathrm{L}$. Thus, diphenhydramine $50 \mathrm{mg}$ IV and hydrocortisone $100 \mathrm{mg}$ IV were administered. Fifteen minutes after the onset of hypotension, BP was restored with $\mathrm{SpO}_{2}$ of $97 \%$, while PIP decreased gradually to $22 \mathrm{mmHg}$. Simultaneously, lung sounds recovered and the capnogram normalized with an $\mathrm{EtCO}_{2}$ of $37 \mathrm{mmHg}$. Surgery continued without unusual findings and ended 7 hours after the anaphylactic reaction. Facial erythema resolved spontaneously within 4 hours. On completion of surgery, the patient's trachea was extubated uneventfully. After being transported to the ICU, the patient's vital signs were stable and he did not complained of respiratory discomfort. However, he had mild pruritus on the trunk and diphenhydramine was administered for 3 days. He was discharged after 3 weeks of hospitalization.

\section{Discussion}

There is still debate as to the proper definition of the term 'anaphylaxis'. A change in terminology was suggested by the World Allergy Organization, which proposed that anaphylaxis refers to a 'severe, life threatening, generalized or systemic hypersensitivity reaction.' They further suggested the term 'allergic anaphylaxis' to be used when this reaction is mediated by an immunologic mechanism and that anaphylaxis from a non-immunologic mechanism should be termed 'nonallergic anaphylaxis.' Therefore, the term 'anaphylactoid' has been eliminated [5]. Foods and medications cause most of the anaphylaxis cases for which the cause can be identified, but virtually any agent capable of either directly or indirectly activating mast cells or basophils can cause this syndrome. Anaphylaxis due to drugs can be caused either by IgEdependent events or the IgE-independent reactions. The mechanisms are different, but clinically indistinguishable and unpredictable [6].

Anaphylaxis is diagnosed with high likelihood based on clinical criteria [5]. These criteria are fulfilled with clinical signs and symptoms onset after allergen exposure, involving the skin or mucosal tissue, plus either respiratory difficulty or a low BP. Traditionally, apart from its clinical features, serum tryptase, plasma histamine and 24-hour urinary histamine metabolites have been clinically used to confirm the diagnosis of anaphylaxis. Other diagnoses that may indicate symptoms similar to anaphylaxis should be excluded. Skin tests and allergen-specific IgE tests can provide confirmatory evidence of sensitization to a specific allergen. In general, skin tests appear to be more sensitive, but less specific than in vitro allergenspecific IgE tests [5].

During general anesthesia, hypotension followed by bronchospasm is the first manifestation of anaphylaxis. In the patient undergoing anesthesia, bronchospasm occurs in less 
than half of cases and skin and mucosal presentations may be late or obscured [7,8]. After all, especially during general anesthesia, increased awareness is very important, because the early symptoms cannot be taken directly from the patient, and other administered anesthetics, as well as the surgical situation, might confuse the differential diagnosis.

In the case presented here, facial erythema was the very first sign, and was too severe and dominant to suspect the allergic reaction. Thus, the subsequently developed bronchospasm, in addition to hypotension, was helpful in the diagnosis of anaphylaxis. Although a cause-effect relationship could not be proven, the absence of any other IV medications or exposure to other potential allergens in the 70 minutes prior to the event, and also development of typical signs within minutes of HES exposure, strongly suggested the association with HES. While this case fulfilled the clinical criteria for diagnosing anaphylaxis, laboratory tests, including serum tryptase, plasma histamine, and 24-hour urinary histamine metabolites should have provided more confirmatory diagnosis for anaphylaxis. Skin tests and allergen-specific IgE measurement could also be useful for identification of the allergen, but the patient refused those tests.

Increased awareness and early, aggressive therapy with vasopressors, steroids and antihistamine injections are essential for the treatment. Vasopressor infusion should be instituted as soon as possible, with titration against both the HR and BP [8].

The physical characteristics of hydroxyethyl starch can be described by their mean molecular weight (MW) and their molar substitution ratio (i.e., the ratio of replacement of glucose by the hydroxyethyl group). The compound Tetrastarch, labelled as $130 / 0.4$, is a low MW product (average $130 \mathrm{kDa}$ ), with a lower molar substitution ratio (0.4). It has been shown that the plasma half-life of an HES preparation increases with increasing MW, and high MS prolongs degradation [3].

The risk of life-threatening anaphylactic reactions caused by HES is very low [2]. Both Kannan and Milligan [9] and Ebo et al. [10] described anaphylactic reactions to 200/0.5 pentastarch, McHugh [11] to pentastarch 250/0.45, and Kreimeier et al. [12] to $10 \%$ pentastarch $(200 / 0.5)$. In contrast, no data on the incidence of anaphylactic reactions after IV administration of the HES 130/0.4 are available. However, it is unlikely that the use of the third generation of HES may have resulted in increased anaphylactic potency, because allergic reaction appears to be induced by the substance itself (starch) and not by the modifications of the substrate. Prediction of anaphylaxis caused by HES is difficult to be made only on the basis of history of drug allergy $[2,6]$. And in this presented case, the patient did not have any history of allergy.

In conclusion, anaphylaxis induced by HES is uncommon, and life-threatening anaphylaxis is rare. In addition, the diagnosis of anaphylaxis is difficult to be made under general anesthesia, when various types of medication are simultaneously used. Therefore, it is essential to understand the criteria for the diagnosis and treatment of anaphylaxis, to make a prompt diagnosis, and to provide a proper treatment. Although we could not perform measurement of serum tryptase, plasma histamine or allergen-specific IgE, as well as skin tests, this case was diagnosed as anaphylaxis induced by HES, because of the typical signs with skin rash, hypotension and bronchospasm developed within minutes after initiation of HES, without using any other agent 70 minutes before the event.

\section{References}

1. Niemi TT, Miyashita R, Yamakage M. Colloid solutions: a clinical update. J Anesth 2010; 24: 913-25.

2. Boldt J. Modern rapidly degradable hydroxyethyl starches: current concepts. Anesth Analg 2009; 108: 1574-82.

3. Ganter MT, Hofer CK, Pittet JF. Postoperative intravascular fluid therapy. In: Miller's Anesthesia. 7th ed. Edited by Miller RD, Eriksson LI, Fleisher LA, Wiener-Kronish JP, Young WL: Philadelphia, Elsevier Churchill Livingstone. 2009, pp 2799-800.

4. Song JG, Eom W, Jeong H, Cho D, Shin HY. Pulseless electrical activity of unknown origin during total gastrectomy. Korean J Anesthesiol 2007; 53: 246-9.

5. Lieberman PL. Anaphylxis. In: Middleton's Allergy: Principles and Practice. 7th ed. Edited by Adkinson NF Jr, Busse WW, Bochner BS, Holgate ST, Simons FER, Lemanske RF Jr: Philadelphia, Mosby Elsevier. 2008, pp 1027-47.

6. Kemp SF, Lockey RF. Anaphylaxis: a review of causes and mechanisms. J Allergy Clin Immunol 2002; 110: 341-8.

7. Mertes PM, Laxenaire MC. Allergic reactions occurring during anaesthesia. Eur J Anaesthesiol 2002; 19: 240-62.

8. Hepner DL, Castells MC. Anaphylaxis during the perioperative period. Anesth Analg 2003; 97: 1381-95.

9. Kannan S, Milligan KR. Moderately severe anaphylactoid reaction to pentastarch $(200 / 0.5)$ in a patient with acute severe asthma. Intensive Care Med 1999; 25: 220-2.

10. Ebo DG, Schuerwegh A, Stevens WJ. Anaphylaxis to starch. Allergy 2000; 55: 1098-9.

11. McHugh GJ. Anaphylactoid reaction to pentastarch. Can J Anaesth 1998; 45: 270-2.

12. Kreimeier U, Christ F, Kraft D, Lauterjung L, Niklas M, Peter K, et al. Anaphylaxis due to hydroxyethyl-starch-reactive antibodies. Lancet 1995; 346: 49-50. 